\title{
To screen or not to screen?
}

\author{
Ngoc-Thy Dinh
}

University of Ottawa

Screening mammography has long been accepted in the western world as an effective public health method for secondary prevention of breast cancer ${ }^{1}$. Currently, Canadian women participate in screening either through an organized program or opportunistic screening ${ }^{1}$. Organized screening occurs within a program where an eligible woman, based on her age and other risk factors, may refer herself directly for mammography ${ }^{1}$. Opportunistic screening occurs when a woman is referred by her family physician to obtain a mammogram ${ }^{1}$. This article aims to highlight the current controversies surrounding screening mammography and important considerations for screening recommendations in Canada.

Since its inception, screening mammography for masses has received little opposition from the general public. However, discussions surrounding the true benefits and harms of such screening have emerged over time. Advancements in treatment, reduction in risk factors (such as use of hormone replacement therapy), and more women taking control over their individual health has resulted in improved breast cancer survival ${ }^{2}$. Recently, a study by Kalager et al. ${ }^{3}$ reported a $10 \%$ reduction in breast cancer mortality attributable to mammography screening. This was a disappointing result according to the authors who expected a reduction of $20 \%$ or more. Other researchers have publicly denounced populationbased mammography screening based on certain claims of harms outweighing benefits, including excessive use of lumpectomies, mastectomies, and radiotherapy, high rate of false positive tests, and over-diagnosis ${ }^{4-7}$.

In the fall of 2009, the U.S. Preventive Task Force updated their mammography screening guidelines by advising screening on a biennial basis for women aged 50-64 only ${ }^{8}$. This garnered much displeasure among women's groups who have argued that women aged 40-
49 should also be screened, despite a lack of evidence for success or cost-effectiveness to support screening for this age group ${ }^{9}$. The reality is that screening is effective in reducing breast cancer mortality in countries that have relatively high disease incidence, including Canada. A $10 \%$ reduction $^{2}$ in disease-related mortality is a considerable benefit. The question that still remains, however, is whether this magnitude of effect is worth the associated costs. Trade-offs between the benefits, harms, and costs associated with various screening guidelines should be considered when making recommendations for routine screening. As previously mentioned, screening younger women (under the age of 50) has not been found to be as cost-effective as screening older women ${ }^{9}$. There is also an issue of resource capacity; a recommendation in which more women are to be screened on a more frequent basis will increase backlog and result in longer wait-times for all women, including those who are at increased risk. Consequently, the mainstream media has used these findings to propagate a concern that mammography screening may not be as beneficial as previously thought and is potentially harmful ${ }^{10,11}$.

Over time there have been a number of important shifts in the way women are screened within organized programs in Canada, and these policies vary regionally. For instance, the program in British Columbia actively screens women on self-referral who are aged 40-49 annually, and women aged 50-79 biennially ${ }^{1}$. This province also accepts women under 40 , provided that they have a referral from a physician. In contrast, Ontario only actively screens women aged 50-74 on a biennial basis ${ }^{1}$. In addition, some provinces are phasing out the use of analog or film mammography in favour of digital mammography, which has been found to be more sensitive in picking up true cancers as opposed to false positives (suspected cancers after screen that are negative at diagnosis $)^{12}$. These varying policies have 
significant impacts on a number of outcomes, including the ability for a program to obtain adequate coverage of the at-risk population, wait-times, and costs related to screening, diagnosis, and treatment ${ }^{13}$.

The pertinent concern that needs to be addressed is why there is so little consensus around population-based mammography screening. This is most likely due to the lack of strong evidence available to support the current practices in terms of effectiveness and efficiency. Other considerations include the assessment of the potential impact of longer screening intervals for women of moderate risk, such as screening every three years, or the impact of tailored screening for women at high-risk. The high-risk category would be comprised of women according to age, as well as family history and/or genetic predisposition. We must also consider the impact of screening vulnerable sub-groups of the population, including women with mental and physical disabilities who face challenges with not only accessing preventive care, but also accessing the health care system in general. Within the context of a publically-funded health care system, decisions regarding which services should or can be funded, and by how much, are particularly difficult to make. To date, there have been very few studies that assess the efficiency or cost-effectiveness of population-based mammography screening in Canada. Decision-makers require sound evidence to support these difficult choices and therefore it is essential that we do not accept the current state of affairs and justify activities based on what has been done in the past. Rather, time should be invested to periodically evaluate these programs to ensure that the benefits outweigh the harms, and that the related costs are reasonable or within society's willingness to pay.

\section{References}

1. Public Health Agency of Canada. Organized breast cancer screening programs in Canada, 2003 and 2004 report. Ottawa: Minister of Public Works and Government Services Canada; 2008.

2. Canadian Cancer Society and National Cancer Institute of Canada. Canadian Cancer Statistics 2008. Toronto: Canadian Cancer Society and National Cancer Institute of Canada, 2008.

3. Kalager M, et al. Effect of Screening Mammography on Breast-Cancer Mortality in Norway. N Engl J Med 2010;363:1203-10.

4. Wright C. Screening mammography and public health policy: the need for perspective. Lancet 346:29, 1995.

5. Olsen O, Gotzsche P. Cochrane review on screening for breast cancer with mammography. Lancet 358:1340-2, 2001 .
6. Horton R. Screening mammography -- an overview revisited. Lancet 358:1284-85, 2001

7. Gotzsche PC, Olsen O. Is screening for breast cancer with mammography justifiable? Lancet 2000; 355:129-134.

8. U.S. Preventive Services Task Force. Screening for breast cancer: U.S. Preventive Services Task Force recommendation statement". Ann. Intern. Med. 151 (10): 716-26, W-236. November 2009.

9. Rosenquist CJ and Lindfors KK. Screening mammography in women aged 40-49 years: analysis of cost-effectiveness. Radiology 191: 647-650, 1994.

10. Salahi, L (2010, September 23). Not as Many Lives Saved by Mammogram, Study Finds. ABCNews, Health, http://abcnews.go.com/Health/OnCallPlusBreastCancerNe ws/breast-cancer-survival-mammograms-livessaved/story? $\mathrm{id}=11713125$ (accessed on February 15 , 2011).

11. Kolata, G. (2010, September 22. Mammograms' Value in Cancer Fight at Issue. New York Times, Health, http://www.nytimes.com/2010/09/23/health/research/23ma mmogram.html?_r=1 (accessed on February 15, 2011).

12. Pisano ED, Gatsonis C, Hendrick E, Yaffe M, Baum JK, Acharyya S, Conant EF, Fajardo LL, Bassett L, D'Orsi C, Jong R, Rebner M; Digital Mammographic Imaging Screening Trial (DMIST) Investigators Group. Diagnostic performance of digital versus film mammography for breast-cancer screening. N Engl J Med. 353(17):1773-83, 2005.

13. Güneş ED, Chick SE, Akşin OZ. Breast cancer screening services: trade-offs in quality, capacity, outreach, and centralization. Health Care Manag Sci. 7(4):291-303, 2004.

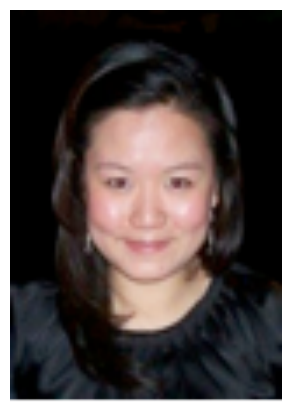

\section{Author Profile}

Ngoc-Thy is a doctoral candidate in Population Health at the University of Ottawa. She holds a Master of Science degree in Community Health and Epidemiology from Queen's University and a Bachelor of Health Sciences Honours degree from McMaster University. In 2008, Ngoc-Thy received a two-year Canadian Cancer Society Research Institute Studentship for her PhD dissertation work, which aims to identify optimal policies for mammography screening in Canada. Ngoc-Thy is a part-time professor for the Interdisciplinary School of Health Science at the University of Ottawa. She currently lives in Ottawa, Ontario and is employed by the Leeds, Grenville and Lanark District Health Unit as the planning and evaluation consultant. 